\title{
Leptin receptor expression in hindbrain Glp-1 neurons regulates food intake and energy balance in mice
}

Michael M. Scott, Kevin W. Williams, Jari Rossi, Charlotte E. Lee, and Joel K. Elmquist

Division of Hypothalamic Research, Departments of Internal Medicine and Pharmacology, The University of Texas Southwestern Medical Center, Dallas, Texas, USA.

\begin{abstract}
Leptin is an adipose-derived hormone that signals to inform the brain of nutrient status; loss of leptin signaling results in marked hyperphagia and obesity. Recent work has identified several groups of neurons that contribute to the effects of leptin to regulate energy balance, but leptin receptors are distributed throughout the brain, and the function of leptin signaling in discrete neuronal populations outside of the hypothalamus has not been defined. In the current study, we produced mice in which the long form of the leptin receptor (Lepr) was selectively ablated using Cre-recombinase selectively expressed in the hindbrain under control of the paired-like homeobox 2b (Phox 2b) promoter (Phox 2b Cre Leprflox/flox mice). In these mice, Lepr was deleted from glucagonlike 1 peptide-expressing neurons resident in the nucleus of the solitary tract. Phox $2 b$ Cre Leprfloxfflox mice were hyperphagic, displayed increased food intake after fasting, and gained weight at a faster rate than wild-type controls. Paradoxically, Phox $2 b$ Cre Leprflox/flox mice also exhibited an increased metabolic rate independent of a change in locomotor activity that was dependent on food intake, and glucose homeostasis was normal. Together, these data support a physiologically important role of direct leptin action in the hindbrain.
\end{abstract}

\section{Introduction}

Leptin, an adipocyte-derived hormone, circulates at concentrations that reflect overall fat mass and informs the body of nutritional status (1-3). Loss of leptin or leptin receptor expression, in both humans and animal models (3-5), leads to a striking elevation in food intake. This is accompanied by decreased sympathetic tone, energy expenditure, locomotor activity, and body temperature that produces marked obesity and alterations in glucose homeostasis. Deletion of leptin receptors in the CNS recapitulates the effects of leptin deficiency on metabolic homeostasis (6). In addition, several studies have found that selective perturbation of leptin action within populations of medial basal hypothalamic neurons accounts for some of the effects of leptin in the regulation of energy balance (7-11). For example, leptin receptor deletion in proopioid melanocortin (POMC) arcuate neurons and steroidogenic factor 1 neurons in the ventromedial hypothalamus (VMH) produces modest obesity $(7,10)$. Interestingly, the metabolic alterations observed in animals with these hypothalamic manipulations of leptin expression were substantially less than those observed in the global receptor null, suggesting that the actions of leptin in the CNS are distributed across neuronal populations in addition to those in the hypothalamus. This is not surprising since leptin receptor expression has been observed in nuclei outside of the medial basal hypothalamus in cell populations implicated in the regulation of food intake and metabolic rate (12-14).

In particular, leptin receptor expression in the nucleus of the solitary tract (NTS) has been suggested to affect food intake through the modulation of meal size as a result of the potentiation of the gastric distention signal received from the vagus (15-18). In rats,

Authorship note: Michael M. Scott and Kevin W. Williams contributed equally to this work.

Conflict of interest: The authors have declared that no conflict of interest exists. Citation for this article: J Clin Invest. 2011;121(6):2413-2421. doi:10.1172/JCI43703. leptin potentiates the NTS response to a gastric preload or to the satiation signal cholecystokinin (CCK), increasing the food intakereducing actions of these stimuli. In agreement with these data, many NTS neurons have been shown to be directly responsive to leptin, resulting in changes in acute neuronal activity $(19,20)$. However, the requirement of leptin action in the NTS in the control of food intake and metabolic homeostasis remains to be established. Both the deletion of leptin receptor and the restoration of receptor expression in specific hypothalamic nuclei have been accomplished using cell type-specific Cre recombinase expressing mouse lines $(7,8,10)$, along with the use of virally delivered recombinase (21) and recombinant leptin receptor (22). In the current report, we describe the use of a transgenic approach in targeting leptin receptor-expressing populations of neurons outside of the hypothalamus, through the development of what we believe to be a novel Cre recombinase expressing mouse line that selectively targets populations of brainstem neurons expressing the transcription factor paired-like homeobox $2 \mathrm{~b}$ (Phox $2 \mathrm{~b}$ ), including glucagon-like 1 peptide (Glp-1) neurons of the NTS (23-26).

\section{Results}

Generation of the Phox $2 b$ Cre mouse line. Phox $2 \mathrm{~b}$ and its paralog Phox $2 \mathrm{a}$ are basic helix-loop-helix transcription factors expressed during embryonic development that are required for the specification of both parasympathetic and sympathetic neurons (24-28). An important aspect of Phox $2 \mathrm{~b}$ expression is the presence of this transcription factor in neurons that form autonomic loops in neurons that form both the afferent and efferent arms of the autonomic nervous system. We predicted that the use of Phox $2 b$ enhancer elements would thus allow for gene expression exclusively in select neurons of the autonomic nervous system. Of interest in the analysis of leptin receptor function, Phox $2 b$ and leptin receptor are both expressed in the NTS $(13,23,24)$. Subsequently, we developed a mouse line expressing Cre recombinase from the Phox $2 b$ locus of a 


\section{Table 1}

Summary of Phox2b Cre-activated lacZ expression

$\begin{array}{lccc} & \text { Line 1 } & \text { Line 2 } & \text { Line 3 } \\ \text { NTS/DMV } & -/+++ & +/+++ & ++/+++ \\ \text { Sympathetic ganglia } & - & - & - \\ \text { Parasympathetic ganglia } & - & - & - \\ \text { Enteric nervous system } & - & - & - \\ \text { Sensory ganglia (DRG) } & - & - & - \\ \text { Nodose } & +++ & +++ & +++ \\ \text { WAT/BAT } & - & - & - \\ \text { Lung, liver, kidney } & - & - & - \\ \text { Testes, thyroid, pancreas muscle } & - & - & -\end{array}$

All lines exhibited high levels of expression in the nodose. Expression in the periphery was absent. All lines showed differences in NTS expression, with line 3 exhibiting the greatest level of expression of Phox $2 b$ Cre lacZ (- indicates no expression, + indicates minimal expression, ++ indicates extensive expression, and +++ indicates expression in all cells). Line 3 was subsequently used to delete Lepr from NTS neurons. DMV, dorsal motor nucleus of the vagus nerve; DRG, dorsal root ganglion; WAT, white adipose tissue; BAT, brown adipose tissue. No expression was observed in the spinal cord (data not shown).

BAC to target the deletion of Lepr from the NTS. Injection of transgene produced several independent founder lines, all of which had variable expression of Cre-activated lacZ in the hindbrain (Supplemental Figure 1; supplemental material available online with this article; doi:10.1172/JCI43703DS1). Importantly, no expression was observed in the hypothalamus (Supplemental Figure 2). Furthermore, transgene expressing neurons also expressed Phox $2 \mathrm{~b}$ as shown by in situ hybridization (Supplemental Figure 1). While all lines showed expression in brachial and visceral motor neurons, along with the sensory ganglia such as the nodose (Supplemental Figures 1 and 2 and Table 1), NTS expression differed markedly. We chose the line with the greatest degree of NTS expression, as evidenced by Cre-activated lacZ expression, line 3 (Supplemental Figure 1). Interestingly, in all founder lines, peripheral expression was absent in the parasympathetic and sympathetic ganglia along with the enteric nervous system. Thus, the Phox $2 \mathrm{~b}$ Cre mouse lines described here appear to be a useful tool in the investigation of gene function in the hindbrain, as they permit hindbrain targeting in either the absence (line 1 ) or presence (line 3 ) of NTS-directed recombination. An initial report of the use of the Phox $2 \mathrm{~b}$ Cre line 2 mice has recently been published, demonstrating the utility of these animals in the selective targeting of the autonomic nervous system (29).

Phox $2 b$ Cre selective deletion of Lepr in the NTS. Phox $2 \mathrm{~b}$ Cre mice were crossed with Leprflox/flox (flox) mice for the analysis of hindbrain leptin receptor deletion. The resulting mice, Phox $2 b$ Cre Leprflox/flox (PC flox) and flox mice, were used in the analysis of hindbrain NTS leptin receptor function. To confirm loss of leptin receptor

\section{Figure 1}

Quantification of Phox2b Cre deletion of Lepr in the hindbrain. Neurons immunoreactive for phospho-STAT-3 were counted after a 12-hour fast and leptin (5 mg/kg) given i.p. (A) Control flox mice showed significantly more NTS STAT $-3^{+}$cells than (B) the PC flox animals. aq, aqueduct. (C) Phox $2 \mathrm{~b}$ Cre deletion resulted in a $60 \%-70 \%$ reduction of phospho-STAT-3 expressing neurons ( $n=4, P<0.02$ ). (D) Glp1 mRNA is expressed by the Phox $2 b$ Cre transgene expressing cells (arrows). Scale bar: $100 \mu \mathrm{m}$ (A and B); $25 \mu \mathrm{m}$ (D). signaling in the NTS, we looked at the ability of leptin to induce phosphorylation of STAT-3 (pSTAT-3), a selective marker of Lepr activity (13). No change in PSTAT-3 in other regions of the brain, including the hypothalamus, was observed. In contrast, a significant decrease of leptin-induced PSTAT-3 was seen in the NTS in PC flox versus flox mice, confirming the selective hindbrain deletion of Lepr (Figure 1). Notably, unlike in the rat (30), wild-type nodose expression of leptin receptor was undetectable (our unpublished observations). This suggests that the leptin receptor deletion in the Phox $2 \mathrm{~b}$ Cre flox animals occurs primarily in the NTS. These data demonstrate the utility of the Phox $2 \mathrm{~b}$ Cre model system in perturbing leptin signaling selectively in the NTS.

Characterization of NTS neurons targeted by Phox $2 b$ Cre. In order to characterize the population of neurons targeted by the Phox $2 b$ Cre transgene, we attempted to identify the neurochemical phenotype of subsets of neurons targeted by the Phox $2 \mathrm{~b}$ Cre transgene. While our prior studies have shown that Lepr-expressing neurons do not coexpress tyrosine hydroxylase (13), we examined Phox $2 b$ Cre-lacZ colocalization with other hindbrain-expressed neuropeptides. Expression of the proopiomelanocortin-derived peptide $\beta$-endorphin was not detected in the hindbrain. However, marked colocalization with Glp-1 was observed (Figure 1D). These data suggest that a primary target of the Phox $2 \mathrm{~b}$ Cre transgene is the Glp-1 neuron, which also expresses leptin receptor.

Hindbrain neuronal Lepr activity defends against weight gain. Several reports suggest leptin may be affecting body weight through actions in the hindbrain $(15-18,31)$. To assess the requirement of Lepr action in the appropriate regulation of body weight, we analyzed weight gain in mice with reduced Lepr expression in hindbrain Glp-1 neurons. Deletion of Lepr in PC flox mice resulted in an increase in rate of weight gain over a 28 -week time period, as determined by analysis of the rate of weight change over time when compared with that of flox controls (Figure 2, $P<0.001$ ). Notably, no difference was observed between wild-type controls and Phox $2 \mathrm{~b}$ Cre mice (Supplemental Figure 3), demonstrating that the Phox2b Cre transgene itself has no effect on body weight homeostasis. Body composition was not altered in 6-week-old mice (Figure 2). At the end of the analysis, there was a trend toward increasing body fat in PC flox mice, while lean body mass was similar between genotypes (Figure 2, $P<0.10$ ). These results reported here are in
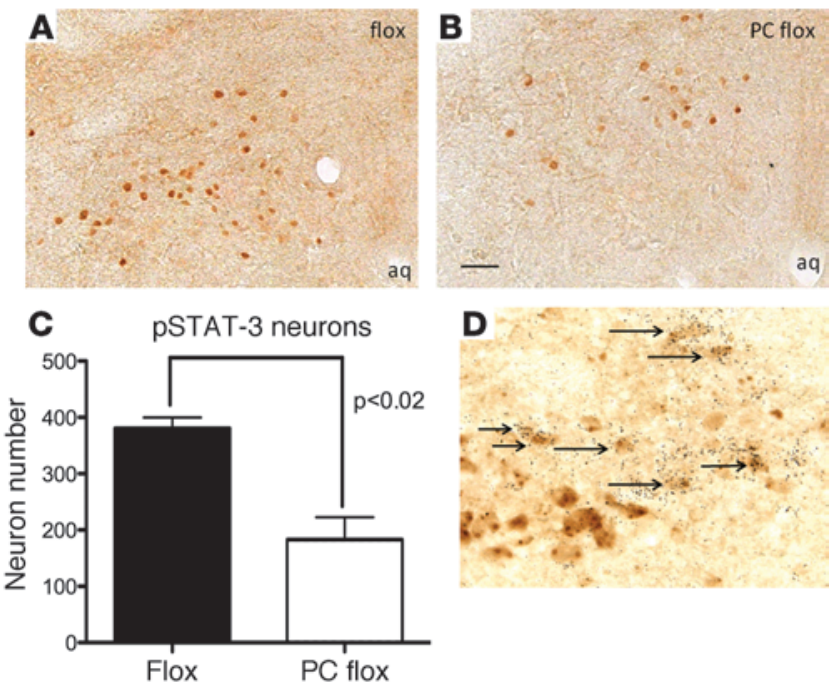
A

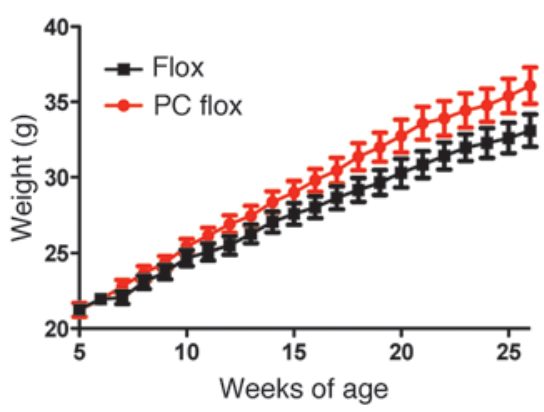

B NMR body composition 6 weeks

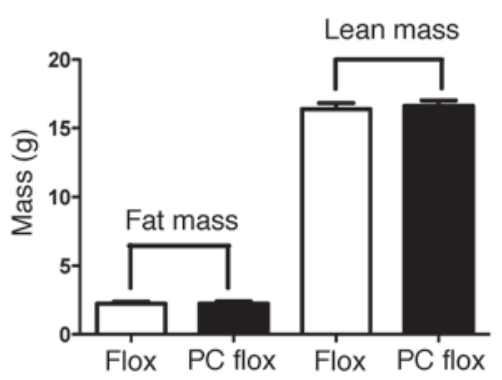

C NMR body composition 28 weeks

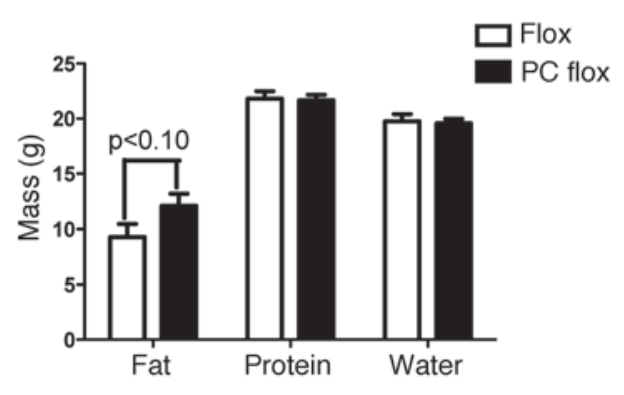

Figure 2

Phox2b Cre deletion of Lepr increases rate of weight gain. (A) Weights of PC flox and flox mice were followed over time on chow diet $(n=20)$. Analysis of the rate of weight changes demonstrated a significant increase in rate of weight gain in the Lepr-deleted mice (95\% confidence interval: PC flox [slope, 0.6095 to $0.7058 \mathrm{~g}$ of body weight per week] versus flox [slope, 0.5089 to $0.5961 \mathrm{~g}$ of body weight per week], $P<0.001$ ). Individual data points suggest a trend toward significant difference in body weight at the end of the study $(P<0.07)$. (B) At 6 weeks of age, no difference in body composition was observed. (C) A trend toward an increase in body fat percentage was observed at the end of the body weight study, with PC flox-deleted mice showing an increase in adiposity $(n=10, P<0.10)$.

agreement with those obtained in the rat using antisense knock down of hindbrain leptin receptor (32). Similar to our data, rat Lepr knockdown (32) resulted in an increase in body weight on regular chow, confirming the role of NTS leptin receptor expression in the regulation of energy balance.

Lepr deletion increases weekly food intake and results in augmented hyperphagia in a fasting-refeeding test. Leptin regulates energy balance in part by the modulation of food intake. It has been suggested that leptin may act in both the hypothalamus and hindbrain to modulate feeding $(7,22)$. To determine the requirement of leptin signaling in the NTS in the regulation of food consumption, intake was monitored in PC flox mice and compared with that in flox controls. Over a 7-day period, mice with a loss of Lepr ate significantly more than controls (Figure 3A). As an independent measure of the action of leptin within the NTS, we next determined the role of leptin action in the control of feeding after an 18-hour fast. Several studies have demonstrated that leptin reduces the amount of food consumed during the refeeding phase (reviewed in ref. 33), illustrating the physiological relevance and potency of leptin in the control of metabolic homeostasis. Following the fast, Lepr-null mice displayed more hyperphagia when refed (Figure 3B), suggesting leptin action is required not only for the regulation of food intake in the fed state but also in the response to fasting as well.

Deletion of neuronal Lepr expression results in an elevation of metabolic rate. The significant alteration in food intake, coupled with only a mild increase in body weight, suggested that metabolic rate may in fact be altered in the PC flox mice and would thus blunt the effect of elevated food intake on body weight. Consistent with this hypothesis, metabolic rate was significantly elevated in the dark phase of the light cycle in hindbrain Lepr-deleted mice. This suggests that the effect of an elevation in food intake resulting from Lepr deletion primarily in NTS neurons is abrogated through the elevation of metabolic rate (Figure 3). The elevation in metabolic rate was unexpected, as prior descriptions of leptin action have cited an increase in metabolic rate upon leptin administration (3). We then examined whether the change in metabolic rate was a primary effect of Lepr deletion or whether it was secondary to the elevation in food intake. Prior to fasting, PC flox mice exhibited an elevated metabolic rate (Figure 4). However, when challenged with an overnight fast, the metabolic rate of PC flox mice was identical to that of the control flox mice (Figure 4). In both the fed and fasted states, locomotor activity was unchanged (Figure 4). The lack of an elevation of metabolic rate upon fasting in the PC flox mice suggests that the altered metabolic rate is dependent on the presence of food and that the elevated metabolic rate is secondary to increased food intake.

We next examined whether NTS leptin receptor deletion modulated the effect of high-fat diet (HFD) on food intake and metabolic rate compared with that in wild-type animals. Interestingly, unlike the phenotype displayed by the chow-fed group, deletion of leptin receptor did not alter chronic body weight homeostasis on HFD (Figure 5). As well, both food intake and metabolic rate were unchanged in PC flox mice relative to those in controls when challenged acutely with HFD feeding (Figure 5).

Normal glucose homeostasis in Phox $2 b$ Cre Lepr-deleted mice. Leptin action on hypothalamic arcuate neurons has been shown to be sufficient for the maintenance of normal glucose levels (21). However, the role of the NTS may also be significant, in that hypothalamic output may influence vagal-dependent glucose production from the liver (33). The requirement of NTS leptin signaling in the control of glucose homeostasis was tested using glucose and insulin tolerance tests. Interestingly, both insulin and glucose tolerance in PC flox mice were identical to those of wild-type mice, suggesting no significant changes in glucose homeostasis (Figure 3). Fasting and fed glucose levels (Figure 3), along with fed insulin levels, were also unchanged in PC flox mice (data not shown) relative to those of controls. These data again demonstrate the distributed actions of leptin, showing how, unlike in the hypothalamus $(7,21)$, leptin acts to affect food intake in the absence of an effect on glucose homeostasis.

Acute responses to CCK injection are intact in mice with Lepr deletion in hindbrain neurons. As noted above, Phox $2 \mathrm{~b}$ Cre deletion of Lepr in the hindbrain produces a significant reduction in leptin-sensing pSTAT-3-positive neurons. To determine whether this reduction is sufficient to blunt the hindbrain sensing of the anorexigenic gut peptide CCK, we injected fasted flox and PC flox mice with CCK-8 $(20 \mu \mathrm{g} / \mathrm{kg})$ and examined the acute anorectic effect of hormone administration. In prior reports, leptin receptor expression has been shown to be required for the actions of CCK in the hindbrain. 
A

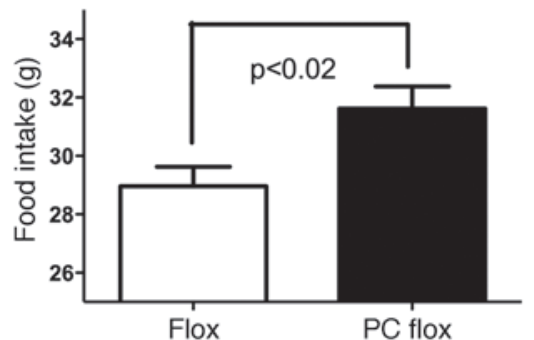

D
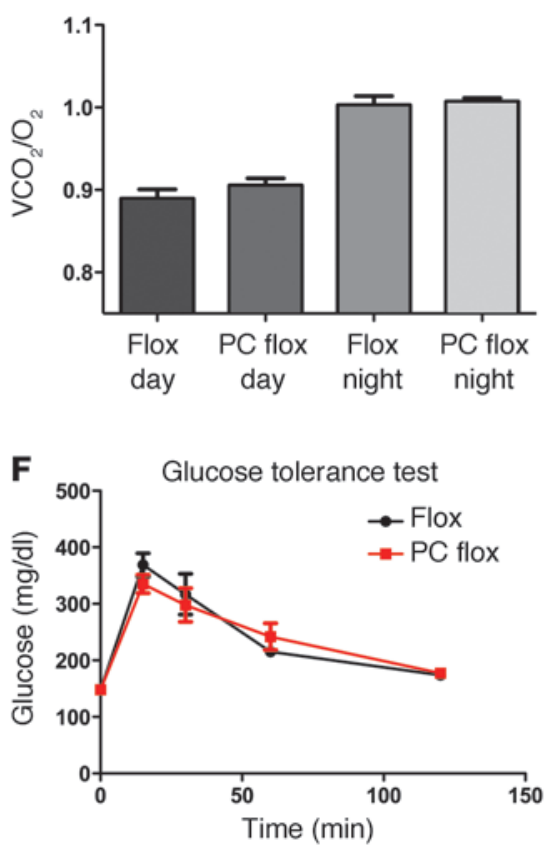

B $\quad 3 \mathrm{~h}$ food intake during refeeding

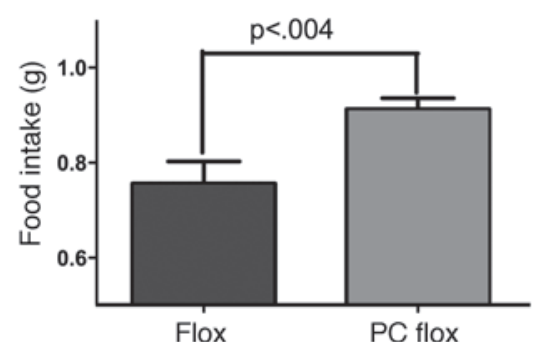

$\mathbf{E}$

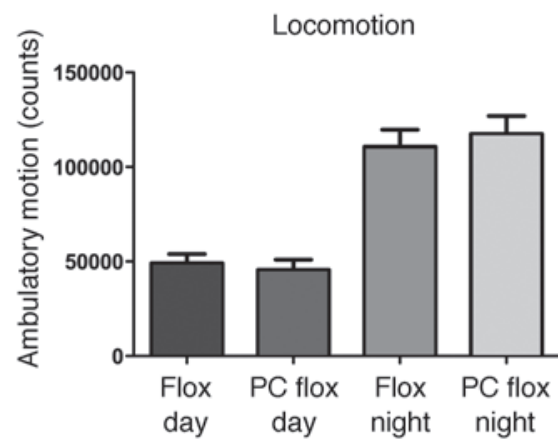

G

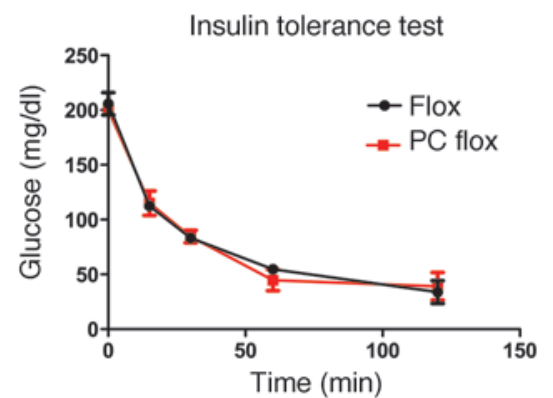

Figure 3

Analysis of food intake, glucose homeostasis, and metabolic parameters. (A) Phox2b Cre deletion of Lepr resulted in an increase in food intake in ad libitum fed mice, (B) while increasing food intake in fasted mice $(n=10)$. (C) An increase in metabolic rate (data were raised to the power of 0.75 ) was observed during the dark phase in the PC flox-deleted mice $(n=10, P<0.05)$. (D) Deletion of Lepr in the NTS produced no alterations in respiratory exchange rate $(n=10)$, indicating that fuel preference was unaltered. (E) No difference in locomotor activity was detected between flox and PC flox mice $(n=10)$. (F) Glucose and $(\mathbf{G})$ insulin tolerance tests showed no difference in glucose handling or in the response to insulin in the Lepr-deleted mice $(n=10)$.

In both PC flox and flox mice, CCK reduced food intake over a 24-hour period (Figure 6), demonstrating that while the number of leptin receptor sensing neurons has been significantly reduced in the PC flox mice, the remaining Leprb expressing cells are capable of responding to CCK (22).

Responses to exogenously applied leptin are intact in the neuronal Leprdeleted mice. We next examined whether mice with deletion of Lepr from NTS neurons respond appropriately to leptin, exhibiting a reduction in food intake and body weight. When control mice received a constant infusion of leptin via osmotic minipump, they exhibited both a reduction in food intake and body weight, relative to those of saline controls (Figure 6). Interestingly, hindbrain Lepr-deleted mice demonstrated a similar reduction in food intake and body weight, suggesting that the hindbrain deletion is not required for the effect of exogenously applied leptin on food intake and body weight.

\section{Discussion}

Much attention has been focused on the actions of leptin in the hypothalamus in the control of metabolic homeostasis. Several studies have demonstrated that leptin action in the arcuate nucleus and VMH (7-9) is relevant to the control of food intake, glucose homeostasis, and metabolic rate. One notable finding from these studies is that only fractions of total leptin deficiency or leptin resistance are recapitulated by neuron-specific deletion of leptin receptors. These findings suggest that the actions of leptin to regulate food intake and energy balance are distributed throughout CNS sites expressing leptin receptors. Consistent with this model, a recent report also suggested that leptin receptors in the hindbrain are required for normal food intake and body weight in rats (32). When leptin receptors in the medial NTS were knocked down, the authors reported a significant 
A
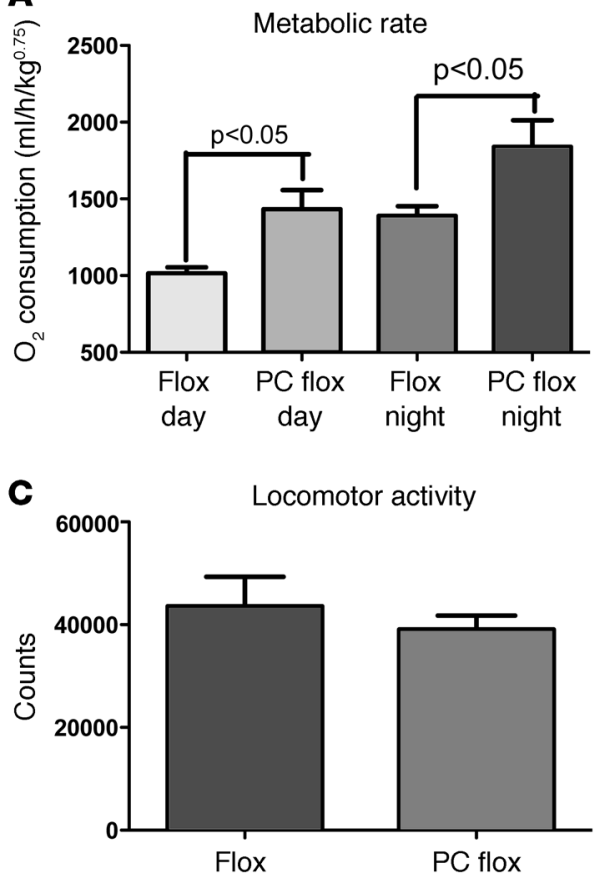

B

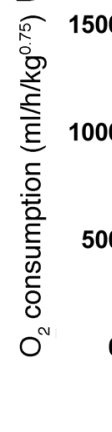

D

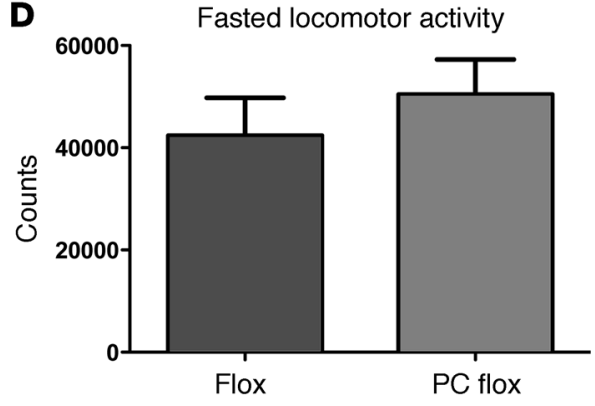

\section{Figure 4}

Elevated metabolic rate of PC flox mice is normalized during fasting. During the initial 3-day period prior to fasting, mice were fed ad libitum. (A) PC flox mice exhibited an elevation in metabolic rate when compared with that of control flox mice. (B) During the fasting period, PC flox mice showed no difference in metabolic rate when compared with that of control flox mice. (C and D) Locomotor activity was unchanged in both groups. increase in body weight and food intake. In this report, we demonstrate that hindbrain leptin receptor action is required for the regulation of food intake, both in the fed state and in response to a fast in mice. Interestingly, unlike in Hayes et al. (32), deletion of leptin receptor, primarily from NTS Glp-1 neurons, also resulted in an increase in metabolic rate that was secondary to the elevation in food intake in the absence of any change in locomotor activity. This response likely contributed to the modest effects of NTS deletion on body weight. Collectively, these results suggest that the actions of leptin in the hindbrain appear to have significant differences from those in other nuclei. For example, the gradual rate of increased weight gain seen with hindbrain deletion was different from that observed in other hypothalamic Lepr deletion models, such as in POMC arcuate cells, in which the deletion produced a modest but more rapid change in body weight before plateauing (7).

Phox $2 b$ Cre and transgenic manipulation of the hindbrain. The generation of the Phox $2 b$ Cre transgenic line has allowed us to reproducibly and selectively target hindbrain NTS neurons, including those expressing Glp-1, leaving other hypothalamic populations intact. Importantly, the lines described in this report target the afferent and efferent arms of the vagus, along with the NTS, permitting a selective targeting of the autonomic nervous system in the absence of peripheral recombination.

The Phox $2 \mathrm{~b}$ transgene, while being expressed in numerous cells of the NTS, does not appear to target all NTS leptin receptor-expressing neurons, as a population remains after Phox $2 \mathrm{~b}$ Cre deletion of Lepr. This may be due to the fact that the transgene is expressed at a low level, resulting in recombination in a select population of neurons. In this case, the transgene would be expected to be expressed in all NTS Lepr neurons. However, the incomplete deletion of Lepr might also result from leptin expression in non-Phox $2 \mathrm{~b}$ expressing NTS neurons, as it is possible that not all NTS neurons express Phox $2 b$. Outside of the NTS, no other site of leptin receptor expression was observed to colocalize with transgene expression. This observation suggests that the observed change in food intake and metabolic rate on chow results solely from the NTS neuronal deletion of Lepr.

Our observation that leptin action in hindbrain NTS neurons of the mouse is required for the regulation of food intake is in agreement with pharmacological and viral approaches aimed at targeting leptin receptor function in the rat. Prior studies have identified NTS neurons that are responsive to gastric distention that are also capable of sensing leptin $(15,17,18)$. These neurons have been shown to express cfos in greater numbers when receiving both gastric input and leptin stimulation. Recently, viral knockdown of leptin receptor in the rat hindbrain confirmed the requirement for leptin receptor signaling in the regulation of feeding, demonstrating the requirement for NTS leptin in the anorectic response to CCK (32). The latter observation differs from our current data, as Phox $2 b$ Cre-mediated deletion of Lepr did not affect the response to exogenously administered CCK. It is quite likely that, since all leptin receptor expression was not completely deleted from the hindbrain by the Phox $2 b$ Cre transgene, the remaining leptin receptor-expressing neurons were still capable of mediating the response to CCK. Alternately, Phox $2 \mathrm{~b}$ Cre deletion of Lepr may have selectively targeted those neurons that are nonresponsive to CCK application while still being responsive to leptin in the regulation of food intake. Similar to this CCK effect, our data demonstrate that hindbrain Glp-1 neuronal Lepr-deleted mice are still capable of sensing leptin. Osmotic minipump delivery of leptin over a 7-day period demonstrated that, while modulation of food intake and metabolic rate requires the full compliment of leptin receptor-expressing neurons of the NTS, other circuits exist that are capable of modulating feeding in response to leptin. Specifically, it is quite likely that leptin receptor-expressing neurons of the NTS that remain in the Phox $2 \mathrm{~b}$ Cre Lepr-deleted animals may be mediating the response 

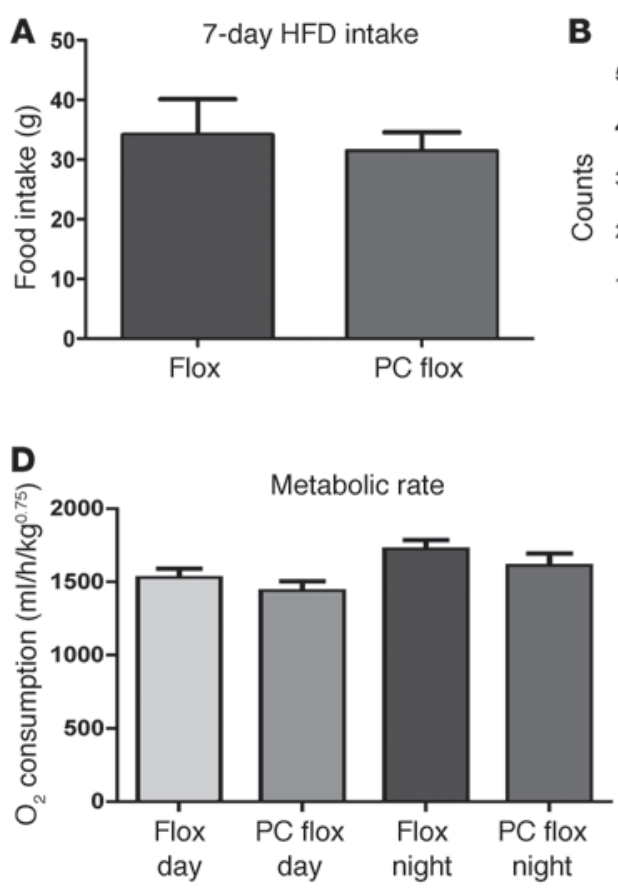

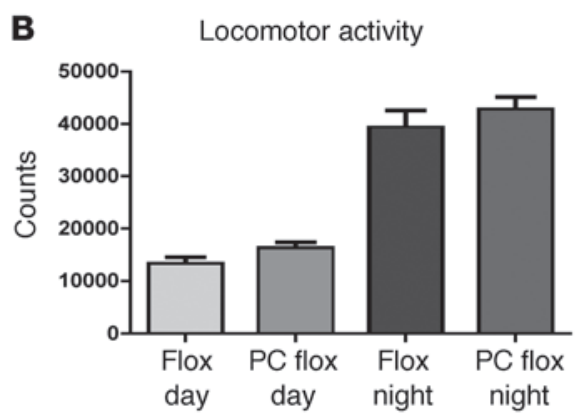

$\mathbf{E}$

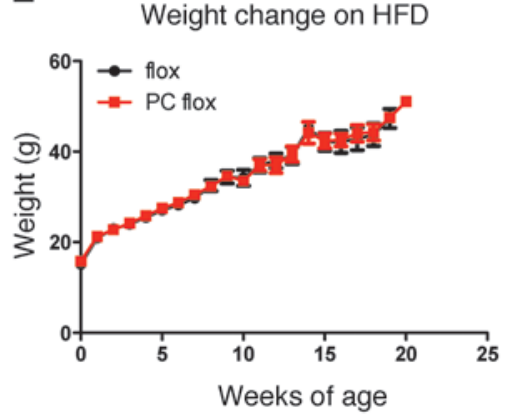

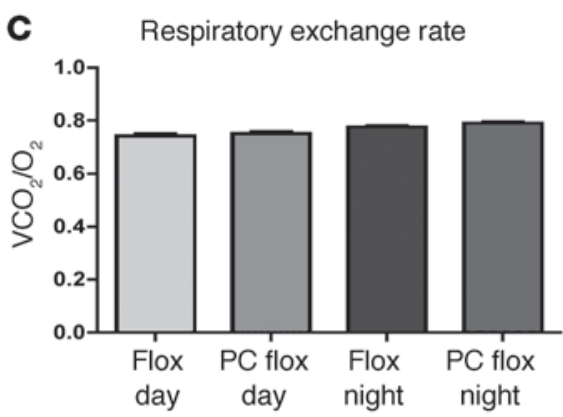

Figure 5

Analysis of food intake and metabolic parameters on HFD. Hindbrain leptin deletion produced no change in (A) food intake, (B) locomotor activity, (C) fuel preference, (D) metabolic rate, or (E) rate of weight change when challenged with HFD-fed diet ad libitum.

to the exogenous dose of leptin. Alternately, leptin applied via the lateral cerebral ventricle may act on another circuit outside of the hindbrain to produce a reduction in feeding and body weight.

The elevation in metabolic rate, observed with the deletion of Lepr in the Phox $2 \mathrm{~b}$ Cre mouse model, was also unexpected. Prior descriptions of leptin action have cited a decrease in metabolic rate upon receptor deletion in the CNS (6). It is then likely that the observed increase in metabolic rate occurs as an indirect result of the elevation in food intake, thereby reducing the hyperphagic effect on body weight gain. The observation that the elevated metabolic rate was present only during the fed state suggests that the ingestion of food was required to manifest the elevation in metabolic rate. The proposed role of NTS Lepr in the elevation of sympathetic outflow, coupled with an elevation in core body temperature (34), also argues against leptin acting to directly suppress metabolism through actions in the NTS. However, further testing of this hypothesis is warranted. For example, a pair-feeding paradigm would permit an analysis of metabolism in the absence of changes in feeding. Such an experiment would thus determine unequivocally whether NTS deletion of Lepr directly affects metabolic rate.

Interestingly, the elevation in metabolic rate may explain the difference in body weight gain observed in this report compared with that of Hayes et al. (32). While we observed a significant change in the rate of body weight gain, Hayes et al. (32) showed a larger effect after leptin receptor knockdown. Differences may be due to several reasons. For example, the extent of leptin recep-
A

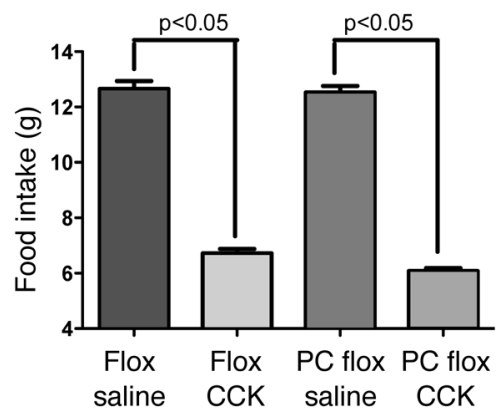

B

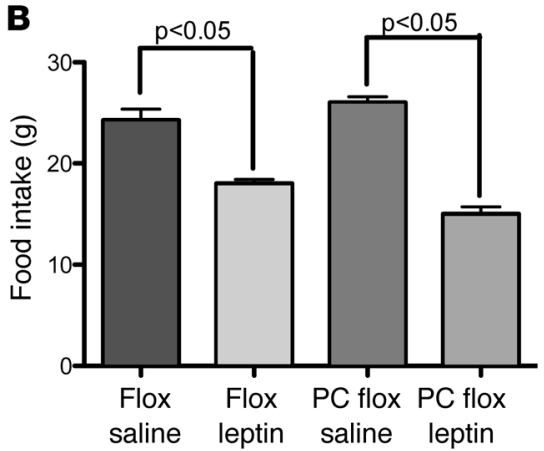

C

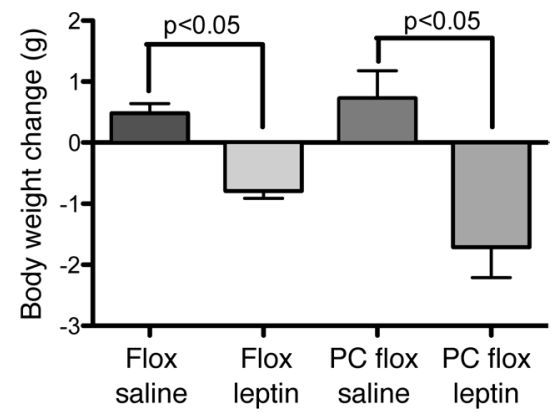

Figure 6

Effect of hindbrain Lepr deletion on CCK- and leptin-induced food intake and body weight. (A) CCK was injected i.p. (20 $\mu \mathrm{g} / \mathrm{kg})$ in PC flox and flox control mice after an overnight fast. Both PC flox and control flox mice consumed significantly less food over a 24-hour period (1-way ANOVA, $P<0.05$ ) compared with that of saline-injected controls. There was no difference between genotypes. Leptin, delivered by osmotic minipump at a rate of $1 \mu \mathrm{g} / \mathrm{d}$ for 7 days, significantly reduced (B) food intake and (C) body weight in both PC flox and control flox mice, relative to that of controls. 
tor deletion differs between our study and that of Hayes. In addition, differences in leptin signaling in the hindbrain of rat and mouse may also exist. For example, in the mouse, nearly half of all hindbrain leptin receptor-expressing neurons express the peptide Glp-1, while, in the rat, coexpression is absent (31). Functional differences between the mouse and rat hindbrain neuroanatomy would then be expected to affect the actions of leptin in modulating metabolism and feeding. This point is especially relevant, as we found that the Phox $2 \mathrm{~b}$ Cre transgene selectively targets the Glp-1 neuronal population.

The lack of effect of Lepr deletion on metabolic homeostasis when challenged both acutely and chronically with HFD was quite surprising. The data suggest that leptin action in sites outside of the hindbrain has a greater modulatory role on regulating food intake and metabolic rate when palatable, calorically dense food is presented. It is also possible that, since a small leptin responsive population of cells remains in the PC flox animals, they would be able to appropriately regulate food intake under conditions of hyperleptinemia during HFD exposure. For example, descending leptin-sensing projections from the hypothalamus might exert a stronger tonic input during high-calorie food intake. Interestingly, while we saw no effect of Lepr deletion in mice fed HFD, Hayes et al. did observe the requirement for hindbrain Lepr in regulating the metabolic changes observed with high-fat feeding. Again, these differences highlight the disparities not only between the rat model used by Hayes et al. and our mouse preparation but also the likely homogeneity of NTS neurons expressing leptin receptor. As the population of NTS neurons targeted by Hayes et al. was not specified, it is possible that our select targeting of glp-1 leptin receptor-expressing cells differed from those neurons targeted by viral injections in the recent manuscript by Hayes et al. Last, there are differences in the timing of hindbrain deletion between our Phox $2 \mathrm{~b}$ Cre model, which has demonstrated gene excision embryonically, and the rat model used by Hayes, which involves gene knockdown during adulthood. Thus, this developmental difference in the timing of leptin receptor perturbation, along with the other methodological and anatomical differences mentioned earlier, might be responsible for the phenotypic differences observed between the 2 models.

Mechanism of NTS Lepr modulation of feeding. The mechanism of hindbrain leptin action in the control of feeding has been hypothesized to involve interactions with hypothalamic neurons that are also leptin sensitive $(22,34)$. Through the sensing of gut stretch, NTS neurons relay information on satiety to hypothalamic centers and receive output from these same areas to produce a change in food intake (35). However, exactly how these pathways interact to produce a change in feeding is only beginning to be described. In prior studies, tracing experiments have shown that the PVN and $\mathrm{DMH}$ receive a significant input from the GLP-1 NTS neurons, demonstrating connections between 2 important hypothalamic sites implicated in the control of food intake (36). Interestingly, output from the NTS Lepr neurons likely involves a polysynaptic loop, signaling first to the hypothalamus and then back to the hindbrain and the vagus, as hindbrain leptin-induced feeding is blocked by a melanocortin antagonist (34).

The action of leptin both in the hindbrain and hypothalamus suggests that there may be redundant sites of leptin action in the control of feeding and that not all sites may be required for the satiating response to leptin. However, the observed change in chow food intake in the Phox $2 \mathrm{~b}$ Cre-deleted mice is significantly greater than any observed effect in the hypothalamus when leptin receptor is deleted from a specific subset of neurons (8). While leptin action in the $\mathrm{VMH}$ was required for the regulation of high-fat food intake (8), the observed changes in food intake in the present study occurred when animals were fed standard chow. This difference highlights the likely divergence in nuclei function in the control of body weight homeostasis, with leptin not being required to affect chow food intake when acting in certain hypothalamic nuclei.

In conclusion, we have shown that leptin signaling in hindbrain neurons is required for the regulation of food intake. In the absence of leptin action in Phox $2 \mathrm{~b}$ neurons, mice consume more and exhibit an elevation in metabolic rate, which likely counters the increase in energy intake, moderating weight gain. These data support and extend findings that leptin effects on food intake require a distributed network of cells, including neurons of the NTS.

\section{Methods}

Animals. All animal procedures were conducted in accordance with The University of Texas Southwestern Medical School's Institutional Animal Care and Use Committee guidelines and those of the Association for Assessment and Accreditation of Laboratory Animal Care. Mice were housed either individually or in groups on a 12-hour-light/dark cycle, with ad libitum access to food and water unless specified otherwise. Floxed leptin receptor (Lepr) mice were backcrossed for 10 generations onto C57BL/6. Mouse colonies were maintained behind a barrier facility and were free of known pathogens. For the experiments described here, Phox $2 \mathrm{~b}$ Cre mice on a C57BL/6 background were initially mated to homozygous flox mice. Hemizygous Phox $2 \mathrm{~b}$ Cre and heterozygous flox offspring were then intercrossed to produce study animals.

Flox mice were genotyped according to prior reports (6). R26R LacZ reporter mice were genotyped using primers, wild type (GCGAAGAGTTTGTCCTCAACC), transgene (AAAGTCGCTCTGAGTTGTTAT), and wild type reverse (GGAGCGGGAGAAATGGATATG), with an annealing temperature of $60^{\circ} \mathrm{C}$. Phox $2 \mathrm{~b}$ Cre mice were genotyped using primers, Phox $2 \mathrm{~b}$ forward (CCGTCTCCACATCCATCTTT), Phox $2 \mathrm{~b}$ reverse (CTACGGACTGCTCTGGTGGT), and Cre reverse (ATTCTCCCACCGTCACTACG), with an annealing temperature of $60^{\circ} \mathrm{C}$.

Phox $2 b$ Cre transgenic mice. To target Cre expression to brachial/visceral motor neurons and to those in the NTS, a BAC clone, RP24-95M11, containing the Phox $2 b$ locus flanked by more than $75 \mathrm{~Kb}$ of genomic DNA at both the $3^{\prime}$ and $5^{\prime}$ ends of the gene, was used to drive Cre recombinase expression (Supplemental Figure 1). A Cre recombinase cassette was introduced into the ATG transcription start codon of the Phox $2 b$ BAC, using bacteria-dependent recombination as performed previously (6). Briefly, a Cre expression cassette fused with a gene encoding neomycin was coupled to $90 \mathrm{bp}$ of DNA homologous to the Phox $2 b$ locus 5' of the Phox $2 \mathrm{~b}$ ATG and to DNA directly $3^{\prime}$ of the ATG by PCR. This targeting fragment was then electroporated into recombination-competent bacteria harboring the Phox $2 b$ BAC. Recombinants were identified by positive antibiotic selection. The Phox $2 \mathrm{~b}$ Cre BAC was injected into C57BL/6J mouse pronuclei to produce independent founder lines. Each line was crossed with the R26R LacZ reporter line to map the activity pattern of Cre recombinase. We subsequently selected a Phox $2 \mathrm{~b}$ Cre line that expressed Cre recombinase-activated LacZ in the greatest number of NTS cells (Supplemental Figure 1). In this particular line, Cre-activated lac $Z$ was detected in parasympathetic visceral and brachial motor neurons as expected, given the role of Phox $2 \mathrm{~b}$ in patterning the development of these cell types (24-28). Interestingly, no Cre-activated Lacz expression was observed in the periphery in this particular cell line, although Phox $2 \mathrm{~b}$ expression is observed in peripheral ganglia along with the enteric nervous system (Table 1). 
Immunohistochemistry and in situ bybridization histochemistry. Phox $2 \mathrm{~b}$ Cre mice were crossed with a Rosa26 $\beta$-galactosidase reporter mouse line (stock no. 003309, The Jackson Laboratory) for the detection of Cre expression in the adult. Mice were perfused transcardially with $4 \%$ formalin, and brains and peripheral tissues were sectioned on a freezing sliding microtome. Immunostaining for lac $Z$ was performed using chicken anti- $\beta$-galactosidase (Ab9361-250, Abcam). Detection of Stat-3 phosphorylation, a marker of leptin receptor activation, was performed as described previously (13). Phospho-specific Stat-3 antisera was obtained from Cell Signaling Technologies. Double label fluorescent immunohistochemistry for ChAT and LacZ was also performed as described previously (13), using goat anticholine acetyltransferase (catalog AB144p, Chemicon). In situ hybridization histochemistry was performed as previously described (13). Primers used to amplify template cDNA for the Phox $2 \mathrm{~b}$ in situ hybridization were as follows: 5'-GCCATCCAGAACCTTTTCAA-3' and 5'-GCTCCTGCTTGCGAAACTTA-3'. Glp-1 in situ hybridization experiments were performed as described previously (37).

Energy expenditure and locomotor activity. Weight- and body composition-matched 4- to 6-week-old and 8- to 12-week-old Phox 2b Cre Lepr flox mice were used for metabolic assessment. Four separate cohorts of animals were used to produce the metabolic data, with measurements sorted into 12-hour-light/dark periods. To exclude the possibility that changes in body weight or composition would contribute to energy expenditure measurements, metabolic assessment was done using weight- and body composition-matched mice (38). Two separate cohorts of Phox $2 \mathrm{~b}$ Cre Lepr flox animals were used to produce the metabolic data, with measurements sorted into 12-hour-light/dark periods. Data, where applicable, were also normalized to lean body mass, by raising metabolic data to the power of $\times 0.75$ (or by expressing data on a per animal basis) (38).

Mice were first acclimatized to the metabolic cages and housed individually for 4 days before measurements were taken. Mice were analyzed in the metabolic chambers for 4 days and were provided with food ad libitum. Energy expenditure was measured by indirect calorimetry, while locomotor activity was assessed using an infrared light beam detection system (Labmaster, TSE Systems GmbH). Data was collected using a TSE Labmaster monitoring system (TSE Systems $\mathrm{GmbH}$ ). Locomotor activity and energy expenditure were determined for both the 12-hour-light and 12-hour-dark cycle as well as for the whole 24-hour period. Data ( $n=10$ mice per group) were averaged over the 4-day period of measurement. For the measurement of fasting metabolic rate, measurements of food intake during unrestricted access were made for 2 days prior to food removal. On the third day, food was removed at the onset of the dark cycle and was replaced after 12 hours. Mice were subsequently followed for 24 hours during refeeding.

Body weight and composition analysis. Animals $(n=20)$ were weaned and placed on a normal chow diet or a Western-style HFD ( $40 \%$ fat by kcal) at 4 to 5 weeks of age. Body weight was measured weekly up to 28 weeks for the chow-fed animals, while HFD-fed mice were followed for 20 weeks. Body composition of 6-week-old and 28-week-old ad libitum fed mice was assessed using nuclear magnetic resonance spectroscopy using an NMR spectrometer (EchoMRI).

Glucose and insulin tolerance tests. Mice $(n=10)$ were fasted overnight and were treated with $2 \mathrm{~g} / \mathrm{kg}$ glucose i.p. (Sigma-Aldrich). For the insulin tolerance test, mice were first fasted for 3 hours and then given $1 \mathrm{U} / \mathrm{kg}$ of insulin (Humulin R, Lilly) i.p. Tail vein blood was assayed for glucose at time 0 and then at 15 minutes, 30 minutes, 60 minutes, and 120 minutes after glucose or insulin administration using a One Touch Ultra glucometer (Lifescan).

Food intake and refeeding. Twelve-week-old mice $(n=10)$ were individually housed for 1 week prior to measurement of food intake. Food intake was then quantified over a 7-day period. For the analysis of food intake after fasting, mice were individually housed and then acclimatized to feeding from a plastic petri dish in the bottom of their cage for 3 days. Mice were then fasted overnight and then presented with a pellet of mouse chow. The amount of food eaten was measured after 3 hours.

Acute responses to CCK. Twelve-week-old mice $(n=10)$ were individually housed for 1 week prior to injection of CCK. For the analysis of food intake after fasting and CCK injection, mice were acclimatized to feeding from a plastic petri dish in the bottom of their cage for 3 days. Mice were then fasted overnight, injected with CCK $(20 \mu \mathrm{g} / \mathrm{kg}$ i.p.) (39), and then presented with a pellet of mouse chow. The amount of food eaten along with the change in body weight was measured after 24 hours.

Osmotic minipump infusion of leptin. Minipump studies were conducted as described previously (40). Singly housed mice underwent stereotaxic surgery to implant a cannula into the lateral cerebral ventricle with following coordinates: $0.34 \mathrm{~mm}$ from the bregma and 1-mm lateral and $2.3-\mathrm{mm}$ ventral from the surface of the skull. An osmotic minipump (Alzet) filled either with saline vehicle or leptin (delivering $1 \mathrm{ng} /$ day for 14 days) was implanted subcutaneously and was attached to the cannula using a catheter. Mice were maintained on chow diet and were allowed to recover from the surgery for 7 days. Food intake and body weight were then tracked for 7 days after this recovery period.

Statistical methods and cell counting. Statistical analysis was carried out using GraphPad 5 (GraphPad) software. Phox2b Cre Lepr flox mice were compared with control Lepr flox mice using a 2-tailed Student's $t$ test with a $P$ value of less than 0.05 being considered significant. In all instances, data are presented as mean \pm SEM. A separate experiment (cumulative body weight change over time) was performed to compare Phox $2 \mathrm{~b}$ Cre mice with wild-type mice to ensure that the Cre line had no homeostatic abnormalities. Body weight curves were compared using a linear regression analysis. Cell counts were performed on every fifth section of each mouse brain. Estimates of cell counts were performed using a $\times 20$ objective. The data were not corrected for double counting and was stereological technique not used, due to the fact that cell size and section thickness did not vary between animals. Neurons were determined to be positively labeled by in situ hybridization if the number of silver grains in a specific cell was greater than that of background signal, defined as signal observed in non-Phox $2 \mathrm{~b}$ expressing neurons outside of the NTS within the hindbrain (41).

\section{Acknowledgments}

We would like to acknowledge Giorgio Ramadori and Teppei Fujikawa for their help in conducting the leptin osmotic pump experiments. We would like to thank the Program Project Grant Core and Mouse Metabolic Phenotyping Core at The University of Texas Southwestern Medical Center at Dallas (supported by NIH grants PL1 DK081182 and UL1RR024923). The work was supported by NIH grants to J.K. Elmquist (R01DK53301, R01DK071320, and RL1DK081185). J. Rossi was supported by a grant from the Sigrid Jusélius Foundation, Academy of Finland, and by a grant from the Finnish Cultural Foundation. M.M. Scott was supported by NIH grant 5K99DA024719-02. K.W. Williams was supported by NIH grants K01 DK087780 and F32 DK077487.

Received for publication December 30, 2010, and accepted in revised form March 9, 2011.

Address correspondence to: Joel K. Elmquist, Department of Internal Medicine - Division of Hypothalmic Research, UT Southwestern Medical Center at Dallas, 5323 Harry Hines Blvd., Dallas, Texas 75390-9077, USA. Phone: 214.648.2911; Fax: 214.648.5044; E-mail: joel.elmquist@utsouthwestern.edu. 
1. Zhang Y, Proenca R, Maffei M, Barone M, Leopold L, Friedman JM. Positional cloning of the mouse obese gene and its human homologue. Nature. 1994;372(6505):425-432.

2. Friedman JM, Halaas JL. Leptin and the regulation of body weight in mammals. Nature. 1998;395(6704):763-770.

3. Halaas JL, et al. Weight-reducing effects of the plasma protein encoded by the obese gene. Science. 1995;269(5223):543-546.

4. Montague CT, et al. Congenital leptin deficiency is associated with severe early-onset obesity in humans. Nature. 1997;387(6636):903-908.

5. Tartaglia LA, et al. Identification and expression cloning of a leptin receptor, OB-R. Cell. 1995;83(7):1263-1271.

6. Cohen P, et al. Selective deletion of leptin receptor in neurons leads to obesity. J Clin Invest. 2001; 108(8):1113-1121.

7. Balthasar $\mathrm{N}$, et al. Leptin receptor signaling in POMC neurons is required for normal body weight homeostasis. Neuron. 2004;42(6):983-991.

8. Dhillon $\mathrm{H}$, et al. Leptin directly activates SF1 neurons in the $\mathrm{VMH}$, and this action by leptin is required for normal body-weight homeostasis. Neuron. 2006;49(2):191-203.

9. Chuang JC, et al. A beta(3)-adrenergic-leptin-melanocortin circuit regulates behavioral and metabolic changes induced by chronic stress. Biol Psychiatry. 2010;67(11):1075-1182.

10. Bingham NC, Anderson KK, Reuter AL, Stallings NR, Parker KL. Selective loss of leptin receptors in the ventromedial hypothalamic nucleus results in increased adiposity and a metabolic syndrome. Endocrinology. 2008;149(5):2138-2148.

11. Morton GJ, et al. Arcuate nucleus-specific leptin receptor gene therapy attenuates the obesity phenotype of Koletsky $(\mathrm{fa}(\mathrm{k}) / \mathrm{fa}(\mathrm{k}))$ rats. Endocrinology. 2003;144(5):2016-2024.

12. Elmquist JK, Ahima RS, Maratos-Flier E, Flier JS, Saper CB. Leptin activates neurons in ventrobasal hypothalamus and brainstem. Endocrinology. 1997;138(2):839-842.

13. Scott MM, et al. Leptin targets in the mouse brain. J Comp Neurol. 2009;514(5):518-532.

14. Mercer JG, Moar KM, Findlay PA, Hoggard N, Adam CL. Association of leptin receptor (OB-Rb), NPY and GLP-1 gene expression in the ovine and murine brainstem. Regul Pept. 1998;75-76:271-278.

15. Grill HJ, Schwartz MW, Kaplan JM, Foxhall
JS, Breininger J, Baskin DG. Evidence that the caudal brainstem is a target for the inhibitory effect of leptin on food intake. Endocrinology. 2002;143(1):239-246.

16. Huo L, Maeng L, Bjorbaek C, Grill HJ. Leptin and the control of food intake: neurons in the nucleus of the solitary tract are activated by both gastric distension and leptin. Endocrinology. 2007;148(5):2189-2197.

17. Schwartz GJ, Moran TH. Leptin and neuropeptide $y$ have opposing modulatory effects on nucleus of the solitary tract neurophysiological responses to gastric loads: implications for the control of food intake. Endocrinology. 2002;143(10):3779-3784.

18. Emond M, Ladenheim EE, Schwartz GJ, Moran TH Leptin amplifies the feeding inhibition and neural activation arising from a gastric nutrient preload. Physiol Behav. 2001;72(1-2):123-128.

19. Williams KW, Smith BN. Rapid inhibition of neural excitability in the nucleus tractus solitarii by leptin: implications for ingestive behaviour. JPhysiol. 2006;573(pt 2):395-412.

20. Williams KW, Zsombok A, Smith BN. Rapid inhibition of neurons in the dorsal motor nucleus of the vagus by leptin. Endocrinology. 2007; 148(4):1868-1881.

21. Coppari R, et al. The hypothalamic arcuate nucleus: a key site for mediating leptin's effects on glucose homeostasis and locomotor activity. Cell Metab. 2005;1(1):63-72.

22. Morton GJ, et al. Leptin action in the forebrain regulates the hindbrain response to satiety signals. J Clin Invest. 2005;115(3):703-710.

23. Stornetta RL, et al. Expression of Phox 2 b by brainstem neurons involved in chemosensory integration in the adult rat. J Neurosci. 2006;26(40):10305-10314.

24. Pattyn A, Morin X, Cremer H, Goridis C, Brunet JF. Expression and interactions of the two closely related homeobox genes Phox $2 \mathrm{a}$ and Phox $2 \mathrm{~b}$ during neurogenesis. Development. 1997;124(20):4065-4075.

25. Pattyn A, Morin X, Cremer H, Goridis C, Brunet JF. The homeobox gene Phox $2 \mathrm{~b}$ is essential for the development of autonomic neural crest derivatives. Nature. 1999;399(6734):366-370.

26. Lo L, Morin X, Brunet JF, Anderson DJ. Specification of neurotransmitter identity by Phox 2 proteins in neural crest stem cells. Neuron. 1999;22(4):693-705.

27. Pattyn A, Goridis C, Brunet JF. Specification of the central noradrenergic phenotype by the homeobox gene Phox2b. Mol Cell Neurosci. 2000;15(3):235-243.

28. Dauger $\mathrm{S}$, et al. Phox $2 \mathrm{~b}$ controls the development of peripheral chemoreceptors and afferent visceral pathways. Development. 2003;130(26):6635-6642.

29. Rossi J, et al. Melanocortin-4 receptors expressed by cholinergic neurons regulate energy balance and glucose homeostasis. Cell Metab. 2011;13(2):195-204.

30. Burdyga G, et al. Expression of the leptin receptor in rat and human nodose ganglion neurones. NeuroScience. 2002;109(2):339-347.

31. Huo L, Gamber KM, Grill HJ, Bjorbaek C. Divergent leptin signaling in proglucagon neurons of the nucleus of the solitary tract in mice and rats. Endocrinology. 2008;149(2):492-497.

32. Hayes MR, et al. Endogenous leptin signaling in the caudal nucleus tractus solitarius and area postrema is required for energy balance regulation. Cell Metab. 2010;11(1):77-83.

33. Grill HJ, Hayes MR. The nucleus tractus solitarius: a portal for visceral afferent signal processing, energy status assessment and integration of their combined effects on food intake. Int J Obes (Lond). 2009;33 suppl 1:S11-S15.

34. Skibicka KP, Grill HJ. Hindbrain leptin stimulation induces anorexia and hyperthermia mediated by hindbrain melanocortin receptors. Endocrinology. 2009;150(4):1705-1711.

35. Grill HJ. Leptin and the systems neuroscience of meal size control. Front Neuroendocrinol. 2010; 31(1):61-78.

36. Vrang N, Hansen M, Larsen PJ, Tang-Christensen $\mathrm{M}$. Characterization of brainstem preproglucagon projections to the paraventricular and dorsomedial hypothalamic nuclei. Brain Res. 2007;1149:118-126.

37. Elias CF, et al. Chemical characterization of leptinactivated neurons in the rat brain. J Comp Neurol. 2000;423(2):261-281.

38. Butler AA, Kozak LP. A recurring problem with the analysis of energy expenditure in genetic models expressing lean and obese phenotypes. Diabetes. 2010;59(2):323-329.

39. Silver AJ, Flood JF, Morley JE. Effect of gastrointestinal peptides on ingestion in old and young mice. Peptides. 1988;9(2):221-225.

40. Ramadori G, Gautron L, Fujikawa T, Vianna CR, Elmquist JK, Coppari R. Central administration of resveratrol improves diet-induced diabetes. Endocrinology. 2009;150(12):5326-5333.

41. Williams KW, et al. Segregation of acute leptin and insulin effects in distinct populations of arcuate proopiomelanocortin neurons. J Neurosci. 2010;30(7):2472-2479. 